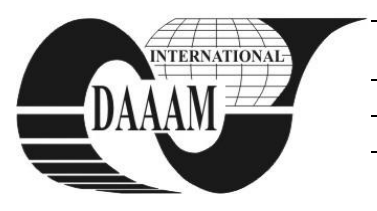

Annals of DAAAM for 2012 \& Proceedings of the 23rd International DAAAM Symposium, Volume 23, No.1, ISSN 2304-1382 ISBN 978-3-901509-91-9, CDROM version, Ed. B. Katalinic, Published by DAAAM International, Vienna, Austria, EU, 2012 Make Harmony between Technology and Nature, and Your Mind will Fly Free as a Bird Annals \& Proceedings of DAAAM International 2012

\title{
CYLINDRIC SHAPE - A DESIGN CREATIVE RESOURCE
}

\author{
MARIN, D[umitru]; RAICU, L[ucian] \& PETRESCU, L[igia] C[armen] M[ariana]
}

\begin{abstract}
This paper presents the role of the cylindrical shape, the most technological and frequent shape in industrial products design. We can achieve different and complex shapes by joining, cutting, developing or by geometrical transformations (rotations, translations, etc.) of cylindrical shape. These new shapes are used because of their geometrical and functional properties differing from the initial shape. At the same time, a special device which turns the ruled cylindrical surface into other ruled surfaces is presented in the paper.

Keywords: geometric shape, surface, technological shape, industrial design, translation, rotation
\end{abstract}

\section{INTRODUCTION}

"Imagination is more important than knowledge" Albert Einstein said. We cannot know if Einstein was totally right, but imagination develops quickly if the man has a lot of knowledge in a certain field of activity. Human being observed what happened around him: the whirlpool, shell's design, spider's web etc., so the helix and the spiral were known since Neolithic. Today, helix and spiral are used in many technique constructions: plane and spatial cams, springs, threads, cutting tools, helical stairs etc.

This example illustrates as clear as possible the inexhaustible resource given by a very old but not obsolete science, namely Geometry $[1,2]$. Geometry is connected with mathematical analysis and algebra. It offers a lot of information about plane and spatial shapes, relations among them, their geometrical transformation, plane and spatial structures which can be achieved by using these shapes, as well as properties and possibilities offered by them. Descriptive geometry is the base of engineering design, the universal language of the technical creation process. It develops intuition and spatial imagination, it represents the scientific base of the technical drawing [3]. The concept of shape appears in almost all definitions for design, given by different schools or personalities [4, 5]. "Design is a creative activity which consists in setting out the shape properties of the industrially made objects. The object shape properties include not only exterior characteristics but also all the structural relations that make a coherent unity from an object or objects system, regarding the viewpoint of either the producer or the end user" (I.C.S.I.D. - The International Council of the Societies of Industrial Design).

\section{CILINDRIC SHAPE AND ITS APPLICATION}

An example, which shows the way the geometric shape is turned into practical application, is given

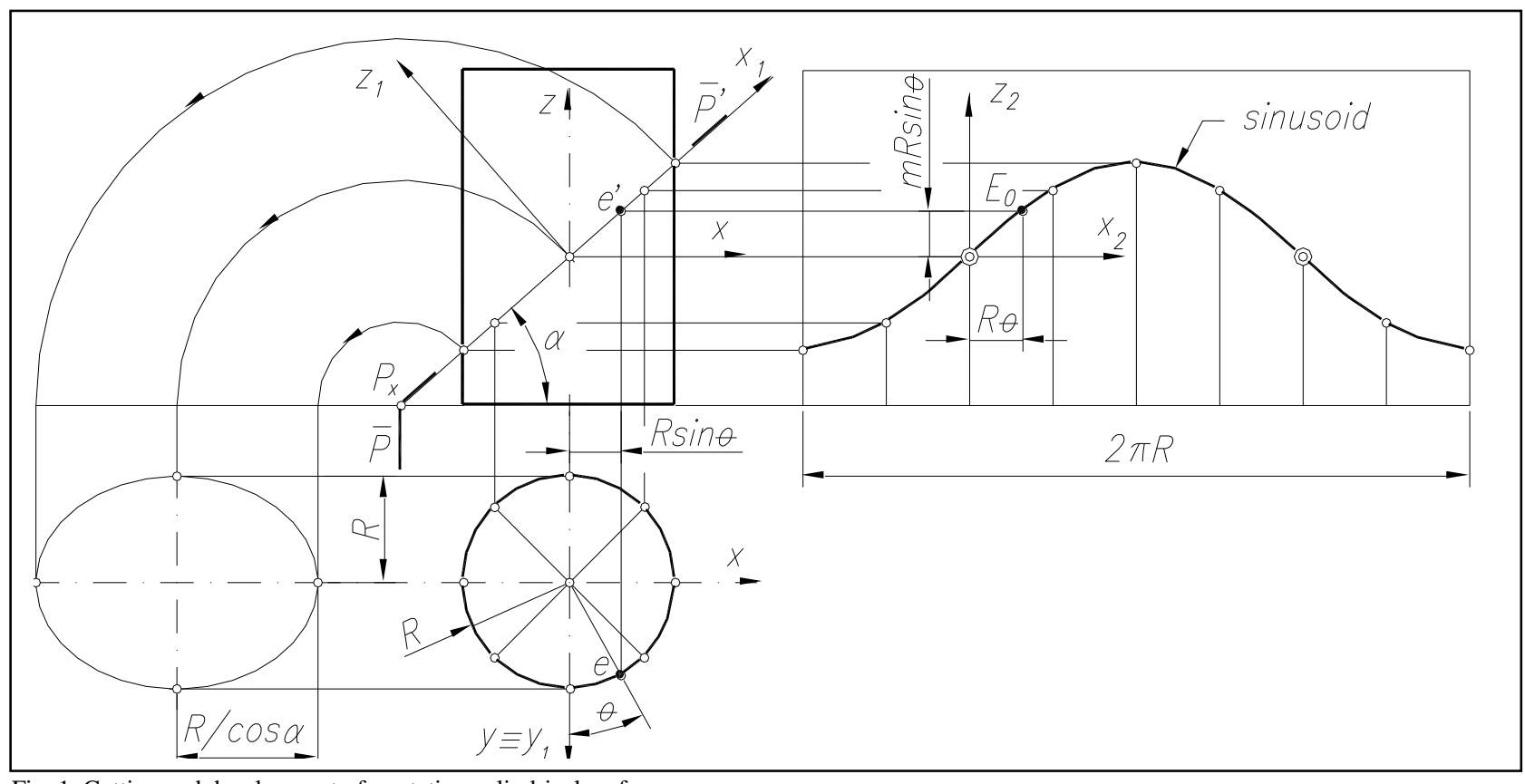

Fig. 1. Cutting and development of a rotation cylindrical surface. 
(Fig. 1).

The followings are proven:

a. The plane section in the rotation cylindrical surface is an ellipse;

b. The development of the section ellipse is a sinusoid. The cylinder equation is:

$$
\left\{\begin{array}{l}
x^{2}+y^{2}=R^{2} \\
z=z
\end{array}\right.
$$

The equation of the $[\mathrm{P}]$ plan is:

$$
z=x \cdot \operatorname{tg} \alpha
$$

The space ellipse equation is:

$$
\left\{\begin{array}{l}
x^{2}+y^{2}=R^{2} \\
z=x \cdot \operatorname{tg} \alpha .
\end{array}\right.
$$

An $\alpha$ angle rotation around the $y$ axis, leads to the plan ellipse equation $\left(x_{1}=x / \cos \alpha ; \quad y_{1}=y ; \mathrm{z} 1=0\right)$, so:

$$
\begin{aligned}
& y_{1}^{2}=\left(x_{1} \cdot \cos \theta\right)^{2}=R^{2} \\
\text { or } & \left(\frac{x_{1}}{R / \cos \alpha}\right)^{2}+\frac{y_{1}^{2}}{R^{2}}=1
\end{aligned}
$$

The coordinates of $\mathrm{E}_{\mathrm{o}}$ on the development are:

$$
x_{2}=R \cdot \theta ; z_{2}=R \cdot \sin \theta \cdot \operatorname{tg} \alpha=m \cdot R \cdot \sin \theta,
$$

Where

$$
m=\operatorname{tg} \alpha=\text { constant. }
$$

In the end,

$$
z_{2}=m \cdot R \cdot \sin \frac{x_{2}}{R} ; \quad \mathrm{x}_{2} \in[0,2 \pi \cdot R] .
$$

The applications of the plane section (ellipse) in the rotation cylinder are shown hereinafter.

1. Elliptic patterns of different sizes obtained by cutting from a cylindrical bar. They are obtained easier than by copying process;

2. Joining the equal diameters pipes to plane (Fig. 2-b) or spatial bends (Fig. 2-c). If sections $\mathrm{S}$ are cut from
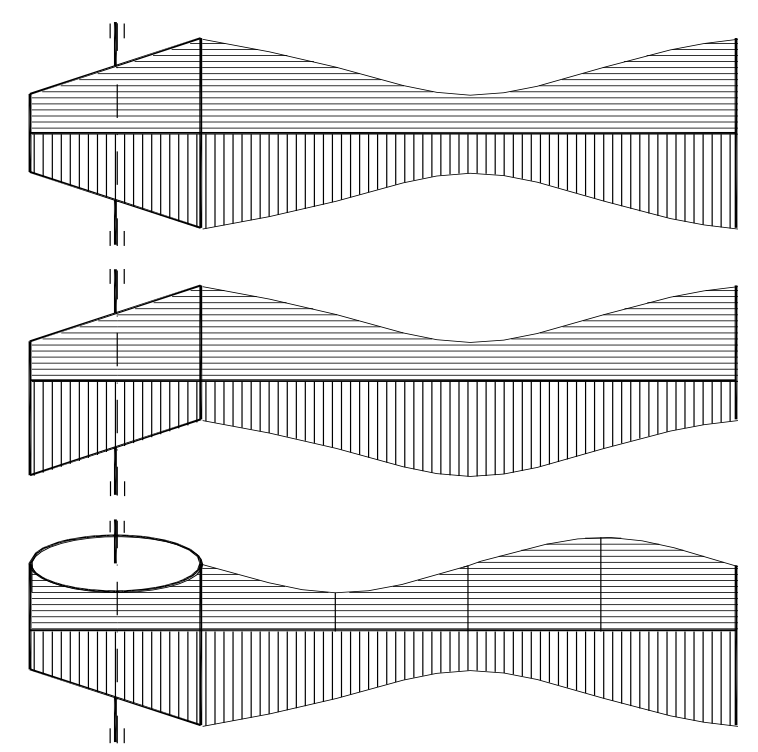

Fig. 3. Plane coverings with different sinusoidal shapes.

the pipe in Fig. 2-a, and are rotated successively at $180^{\circ}$ the plane bend (Fig. 2-b) or an approximate torus segment it can be obtained;

In order to achieve the spatial bend in Fig. 2-c, the connection ellipses between sections are not in mirror any longer but rotated by $\varphi$ angle, meaning, before cutting the pipe after the $E_{2}$ ellipse, this is rotated by the $\varphi$ angle;

3. Precise cutting out of small thickness sheets $(0.2-$ $0.5 \mathrm{~mm}$ ) to a sinusoid, sheets used at pipes joining, tubulatures, etc;

4. Different spatial lines (for example banisters) made of bars or pipes (Fig. 2-d);

5. Plane coverings with different sinusoidal shapes in desired colours and textures by joining the cylindrical sections and rolling them on a plane surface (Fig. 3); In the above mentioned example we used: concepts of plane and spatial geometry; analytical geometry (calculus relations); descriptive geometry (projections, rotations, developments); elements of engineering graphics (drawings, dimensions) and relations among cylindrical sections, in order to set out practical applications.

All these, together with manufacturing technology sections cutting, chamfering, welding and their

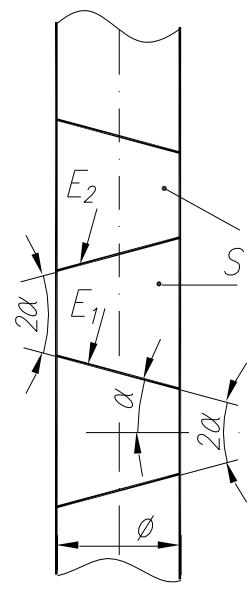

a.

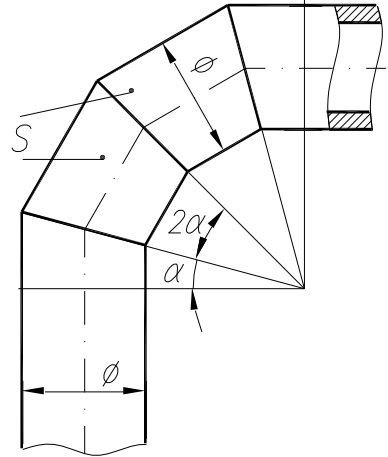

b.

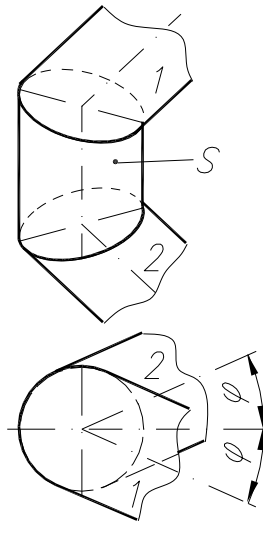

$-0180-$

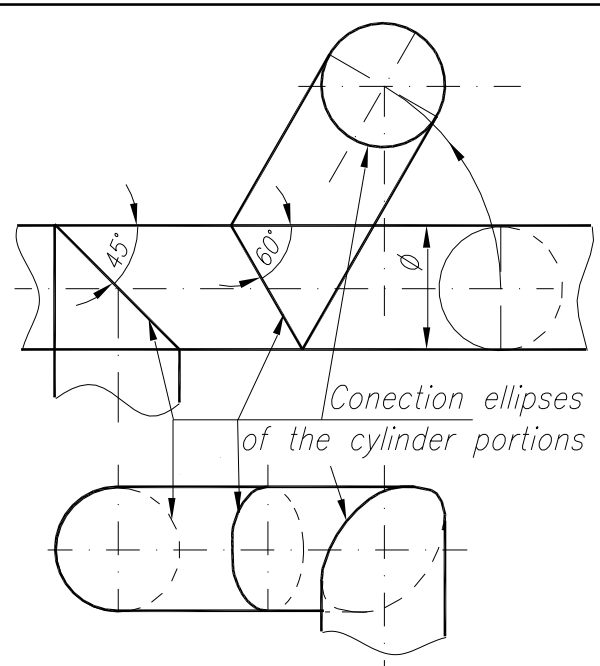

d. 
assembling, represent design work for achieving a useful and at the same time aesthetic product $[2,6]$.

\section{CILINDRIC RULED SHAPE AND ITS APPLICATIONS}

In Fig. 4-a, the rotation cylinder with the base circles $C_{1}$ and $C_{2}$ connected to the $G$ inextensible generatrix by spherical joints is presented [7].

By rotating the $C_{1}$ circle, the generatrix will be in a new position (Fig. 4-b). The cylinder turns into a rotation hyperboloid [8].

The height of the hyperboloid is $H-h$.

A new generatrix position (a front segment) was considered, to become simpler. In the rectangular triangle $b^{\prime} e^{\prime} a_{l}{ }^{\prime}$, we can write:

$$
b^{\prime} e^{{ }^{2}}=b^{\prime} a_{1}{ }^{2}-e^{\prime} a_{1}{ }^{\prime}
$$

or:

$$
(H-h)^{2}=H^{2}-4 \cdot R^{2} \cdot \sin ^{2} \alpha .
$$

Finally, after calculations it is obtained:

$$
h=H-\sqrt{H^{2}-4 \cdot R^{2} \cdot \sin ^{2} \alpha}
$$

The $h$ movement of the $C_{1}$ circle is found to depend on the $H$ and $R$ constant cylinder parameters and the angle $2 \alpha ; 2 \alpha \in(0, \pi)$.

$$
\text { If } \begin{aligned}
2 \alpha=\pi & \\
& \Rightarrow h=H-\sqrt{H^{2}-4 \cdot R^{2}}
\end{aligned}
$$

the cylinder becomes cone, provided that $H>2 \cdot R$.

We can mention as practical applications of this example, the followings:

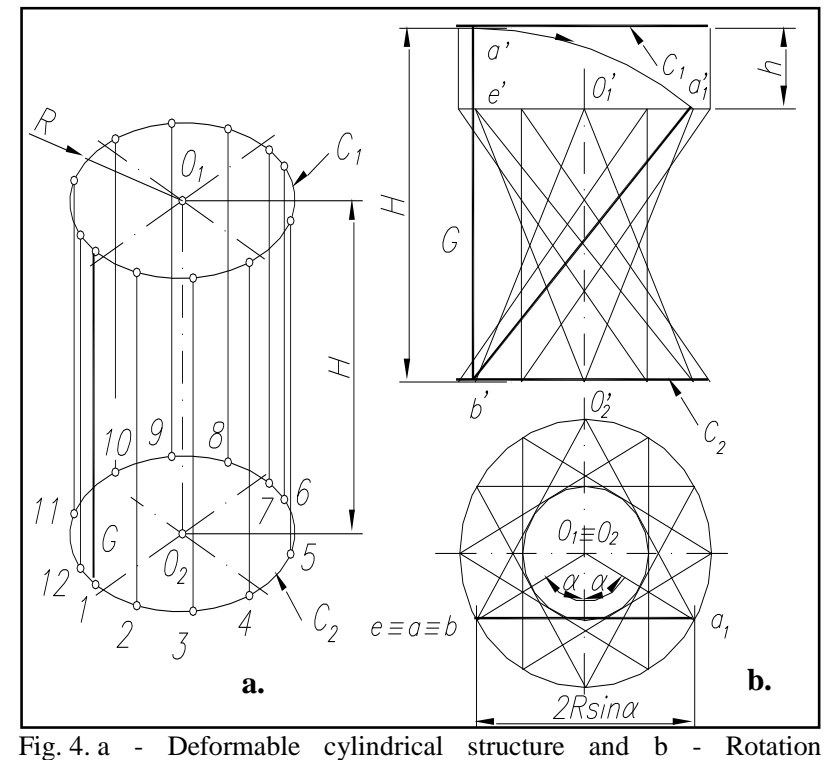
hyperboloid

- Linear and angular movement mechanism made of several hyperboloids connected among them. It is used for stepper robots - it helps the robot lift its legs in order to step [8];
- Clamping, pressing, grasping mechanisms when the hyperboloid generatrixes are rigid bars attached to the $C_{1}$ and $C_{2}$ disks in Fig. 4-b. By rotating one of the disks (the other one is fixed), bars come near and create pressing forces at the level of the girdle circle.At the same time, by rotating and moving one of the disks, axial forces could be created in a direction or another; the cylinder will become a hyperboloid and the distance between disks decreases or the hyperboloid will be turned into cylinder and distance between disks increases (a kind of winch);

- Wheels, rotors, adjustable abrasive or cutting tools based on property of the hyperboloid to change its profile to a cylinder [7];

- Different ornamental items can be obtained, (for example a lamp-shade) which could be changed from a cylinder into many hyperboloid shapes [6].

\section{EXTENSIBLE GENERATRIX CYLINDER AND ITS APPLICATIONS}

In Fig. 5-a, a device which turns the rotation cylinder with extensible generatrix into a lot of other ruled surfaces [9] is presented. The component parts of the device are:

$\mathbf{1}$ - base plate; $\mathbf{2}$ - guiding bar; $\mathbf{3}$ - cylindrical slides; 4 - disks supports; 5 - disks with holes; 6 - extensible threads.

- Oblique circular cylinder in Fig. 5-b is achieved by cylindrical slide 3 turning on guiding bar 2 and by

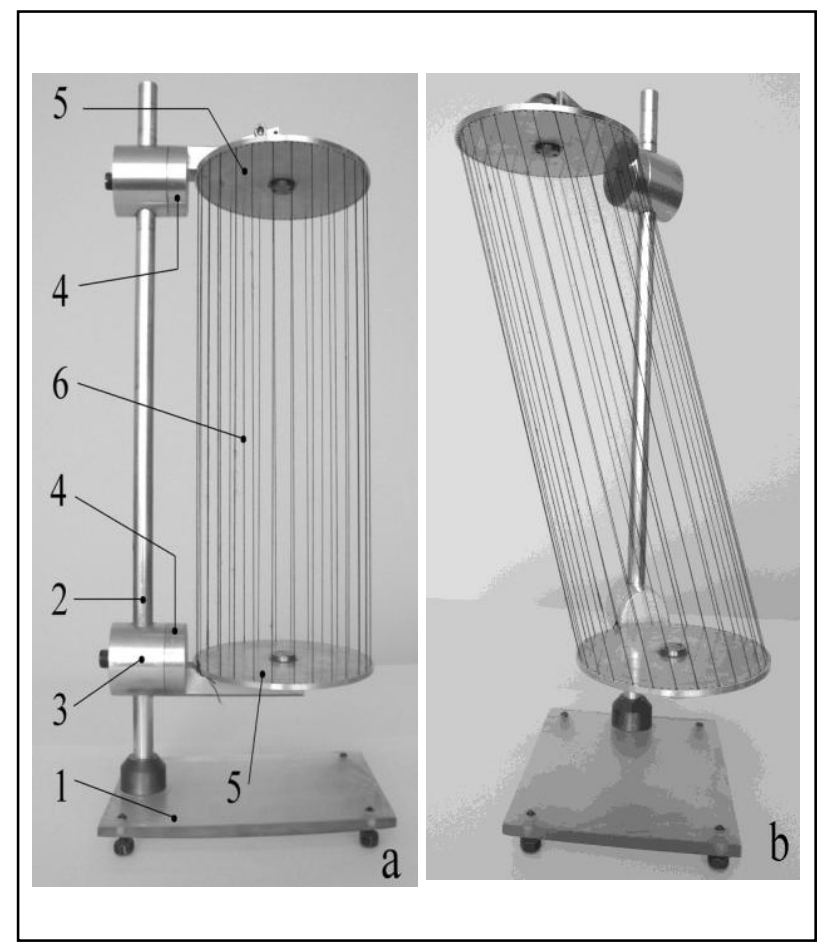

Fig. 5. Cylindrical surfaces: a - rotation cylinder, b - oblique circular cylinder. 


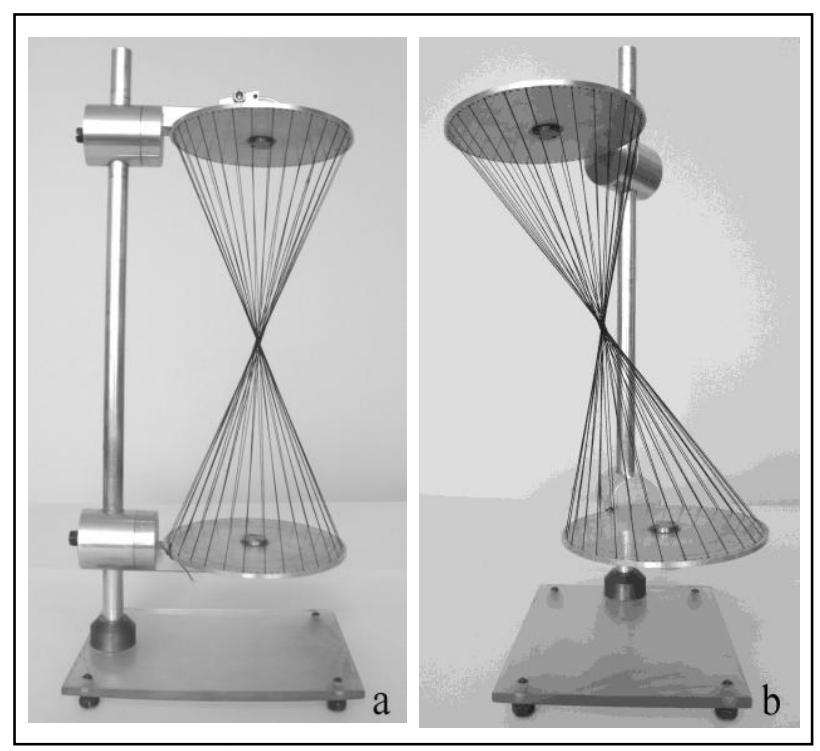

Fig. 6. Conical surfaces: a - rotation cone, b - oblique circular cone

superior disk 5 turning round its own axis, in order for the generatrix to become parallel;

- Rotation cone in Fig. 6-a is achieved by superior disk 5 turning round its own axis with angle $\alpha=\pi$;

- Oblique circular cone in Fig. 6-b is achieved from 6a, by cylindrical slide 3 turning on guiding bar 2 ;

- Rotation hyperboloid in Fig. 7-a is achieved by superior disk 5 turning round its own axis with angle $\alpha<\pi$;

- Oblique hyperboloid in Fig. 7-b is achieved from 7-a by cylindrical slide 3 turning on guiding bar 2 ;

- Straight cylindroid in Fig. 8-a is achieved from 5-a by support 4 turning round its own axis with angle $\alpha<\pi / 2$ or by superior disk 5 turning round its own diameter;

- A certain cylindroid, achieved from 8-a by cylindrical slide 3 turning on guiding bar 2, is presented in Fig. 8-b.

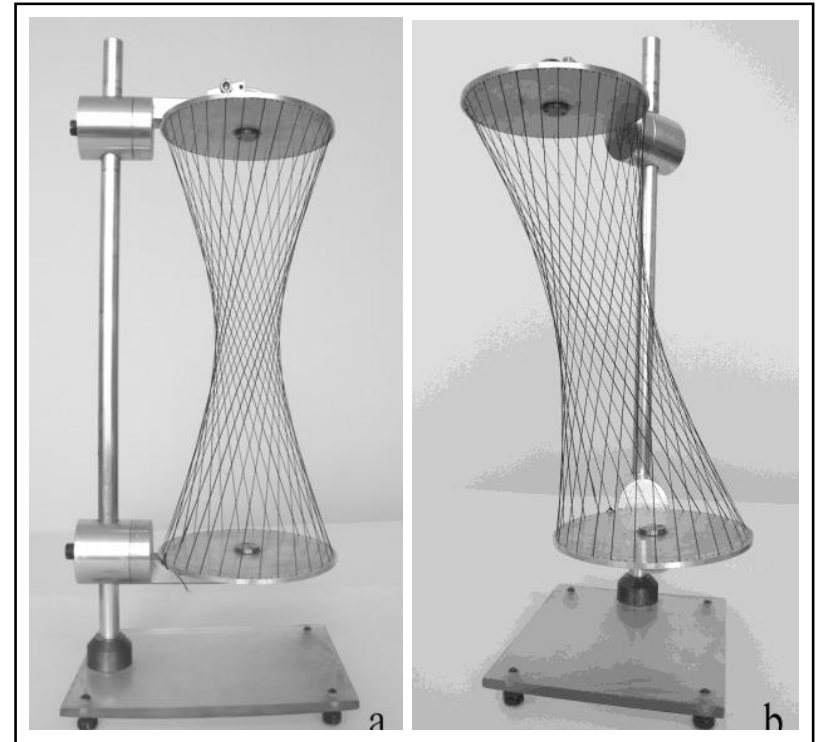

Fig. 7. Hyperboloid surfaces: a - rotation hyperboloid, b - oblique hyperboloid

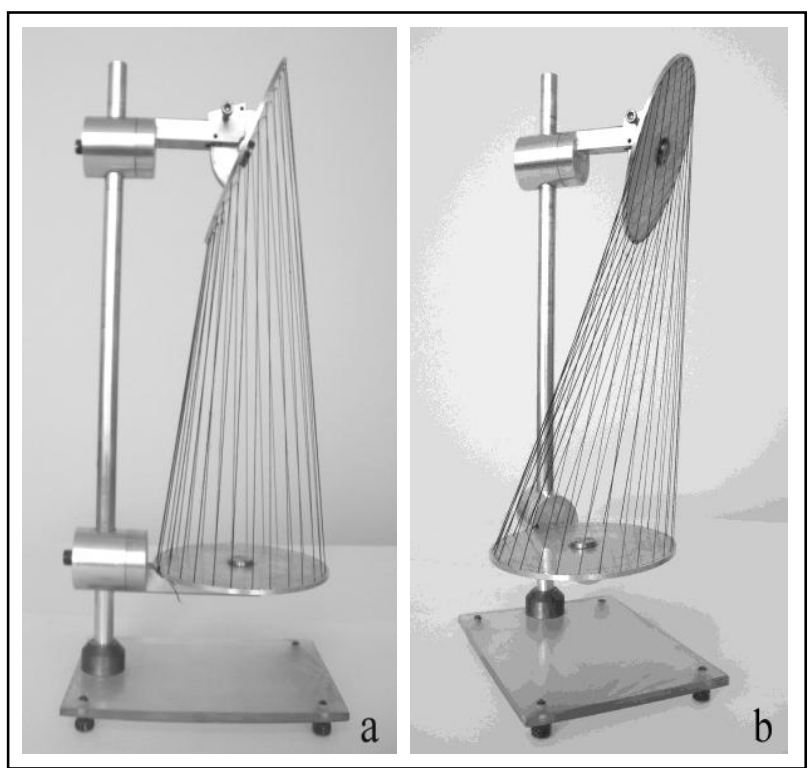

Fig. 8. Cylindroid surfaces: a - straight cylindroid, b - a certain cylindroid

The presented device is an excellent demonstrative material (teching aids, school supplies) but a lot of ornamental items ccould be imagined by using its properties.

\section{CONCLUSIONS}

This paper shows the importance of geometry, generally, and cylindrical shapes, especially, in engineering design. The primary cylindrical shapes but especially their transformations by cutting, developing, wrapping, arranging, rotating or other similar operations, are an inexhaustible creative resource in the design of industrial products. At the same time, the cylindrical surface could be turned into many other surfaces if their generatrixes are extensible, by using a special device.

\section{REFERENCES}

[1] Papanek, Victor, (1997), Design for the real world, Technical Publishing, ISBN 973-31-1019-3, Bucureşti

[2] Raicu, Lucian, (2002), Graphic and visual, between clasic and modern, PAIDEIA Publishing, ISBN 978-606-521-116-2, Bucureşti

[3] Dumitrescu, Andrei, (2000), Design, PRINTECH Publishing, ISBN 973-652-115-X, București

[4] Quarante, Danielle, (1994), Éléments de design industriel, Polytechnica $2^{\mathrm{e}}$ édition, ISBN 2717842330, Paris

[5] Fiell, Ch., Fiell, P. (2005). Design of the $20^{\text {th }}$ Century Taschen GmbH, ISBN 3-8228-4078-5, Köln

[6] Marin, D., (2011), Industrial Design - Shape Design, BREN Publishing, ISBN 978-973-648-706-4, Bucuresti

[7] Plahteanu, B. \& Belous, V. (2000), Geometrical Efects in the Techical Creation, Performantica Publishing, ISBN 973-807501.7, Iasi

[8] Marin, D., Raicu, L. , Simion, I., (2008), The role of the shape in industrial design, The 19th INTERNATIONAL DAAAM SYMPOSIUM, ISSN 1726-96-79, Trnava, Slovacia

[9] Marin, Gh., Petrescu, Ligia, Dolga, Lia, (2008), The cylinders intersections visualization in teaching Descriptive Geometry, The 19th INTERNATIONAL DAAAM SYMPOSIUM, ISSN 1726 96-79, Trnava, Slovacia 